\title{
Effect of Hall current on MHD mixed convection boundary layer flow over a stretched vertical flat plate
}

\begin{abstract}
In this paper, the steady magnetohydrodynamic (MHD) mixed convection boundary layer flow of an incompressible, viscous and electrically conducting fluid over a stretching vertical flat plate is theoretically investigated with Hall effects taken into account. The governing equations are solved numerically using an implicit finite-difference scheme known as the Keller-box method. The effects of the magnetic parameter, the Hall parameter and the buoyancy parameter on the velocity profiles, the cross flow velocity profiles and the temperature profiles are presented graphically and discussed. Investigated results indicate that the Hall effect on the temperature is small, and the magnetic field and Hall currents produce opposite effects on the shear stress and the heat transfer at the stretching surface.
\end{abstract}

Keyword: Stretched flat plate; Hall effect; Magnetohydrodynamic; Mixed convection; Boundary layer 